\title{
Discrimination learning after hippocampal lesions in 1-day-old rats
}

\author{
J. H. BLUE \\ Laboratory of Developmental Psychology \\ National Institute of Mental Health, Bethesda, Maryland 20205 \\ and \\ J. A. COOPER and SHERMAN ROSS \\ Howard University, Washington, D.C. 20059
}

\begin{abstract}
Hooded rat pups $(\mathrm{N}=26)$ received radio frequency lesions at 1 day of age. Ten were operated controls, nine received hippocampal ablations, and seven sustained lesions of the entorhinal area. The effects of the surgery were assessed using a left-right spatial discrimination test with the home cage and mother serving as reward. The results suggest that partial deefferentation of the hippocampus may facilitate performance on a spatial discrimination task.
\end{abstract}

In experimental animals, brain damage occurring in adulthood appears to be more deleterious than brain damage occurring in infancy. Different effects have been found in patients (Hebb \& Penfield, 1940). As proposed by Teuber (1971), the conclusion that only species differences are involved may be deceptive. After reviewing the animal and clinical findings, Teuber suggested that one reason why results obtained at the two phylogenetic levels were incongruent was that too narrow a range of function was being tested in experimental animals. Work with young patients who sustained surgery for relief of epilepsy supports this suggestion. Though it is true that there is a relative escape of language after damage to the left cerebral hemisphere, investigators have been able to demonstrate that this happens at a price in other nonverbal functions normally mediated by the right hemisphere (Milner, 1974). In humans, bilateral damage to the hippocampus, a brain structure in the depths of the temporal lobe in primates and also well developed in rodents (Bayer, 1980), results in severe and permanent disturbances of short-term memory (Penfield \& Milner, 1958). The present study was designed to determine whether similar deficits could be found after ablation of the hippocampal formation in rats at 1 day of age. The behavioral effects of surgery were assessed using a left-right spatial discrimination test, with the home cage and mother serving as reward.

\section{METHOD}

\section{Materials}

Male and female hooded rat pups, born in the rat colony of

These results were secured at Northeastern University, Boston, Massachusetts, prior to the first author's employment at NIMH. Appreciation is extended to Helen Mahut, Martin Block, and Alex Skavenski for help. Requests for reprints should be sent to J. H. Blue, NIMH, LDP, 15-K, 9000 Rockville Pike, Bethesda, Maryland 20205. the Neuropsychology Laboratories of Northeastern University, Boston, Massachusetts, were the subjects. The mothers were impregnated at Blue Spruce Farms in New York. A 12 h light/ $12 \mathrm{~h}$ dark cycle was used. Between 18 and $24 \mathrm{~h}$ after birth, the following types of bilateral surgery were performed: lesions of the entorhinal area $(n=7)$, lesions of the hippocampus $(n=9)$, and operated controls $(n=10)$. The pups were anesthetized by hypothermia by placing them on ice and papers for approximately $90 \mathrm{sec}$ or until all responses to external stimuli had ceased (Schwartz, 1963). The skin above each lesion site was punctured using a No. 23 hypodermic needle. A monopolar electrode was lowered through the hole and radio frequency lesions were made. The intensity of the Grass Instruments lesion maker was 70 , with a duration of $10 \mathrm{sec}$. After surgery, all pups were placed on paper towels over an operating heating pad. A mixture of distilled water, quinine sulfate, and Bacitracin ointment was placed on the heads and backs of the pups to discourage cannibalism by the mother. All pups were active and responsive to tactile stimulation before being returned to the mother and home cage, where they remained for 16 days for recovery. Twenty-four hours before behavioral testing, each pup was removed from its mother and housed in a $31 \times 111 \mathrm{~cm}$ Plexiglas box. The floor was covered with wood shavings over the heating pad. The animals were tested at 17 days of age after a $24 \mathrm{~h}$ separation from the mother. Rats were assigned arbitrarily to the paths (half of the pups to the left side and the other half to the right side). Acquisition and reversal trials were conducted on the same day until a learning criterion of 8 of 10 consecutive correct responses was attained. Each trial lasted a maximum of $60 \mathrm{sec}$. If no responses were made, the pup was placed in a closed wooden box, designated the "isolation box" (Figure 1). Following an incorrect choice, the pup was placed in the isolation box for $30 \mathrm{sec}$. Correct choices were followed by $15 \mathrm{sec}$ access to the goal compartment in contact with the mother. The apparatus (Figure 1) consisted of a small starting box, a choice area, a goalbox, and a home cage (Bulut \& Altman, 1974). The starting box $(9 \times 9 \times 7 \mathrm{~cm})$ opened through a small hole $(3.8 \mathrm{~cm}$ in diameter) into a wooden two-choice discrimination area $(24 \times 30.9 \times 15 \mathrm{~cm})$, which was connected to the goalbox filled with shavings. A perforated Plexiglas wall separated the goalbox from the home cage. A similar perforated partition blocked the entrance of the incorrect side. A T-shaped partition divided the choice area into two sides, allowing entry into the goal area. Immediately after testing, the animals were sacrificed with Metophane perfused through the left ventricle with physiological saline and $10 \%$ formalin.' All brains were removed, fixed, 
sectioned at 40 microns, and stained with cresyl violet. Histological analysis revealed damage to the entorhinal area (Figure 2). Most lesions included the medial aspects of the entorhinal area. Some showed partial ablations of the lateral aspects of the entorhinal cortex. Partial ablations of the hippocampus were noted (Figure 3). Some animals incurred damage to the dorsal and ventral portions of the hippocampus. The differences in the amount of damage in the various groups of animals may be due in part to the previously reported regrowth in the hippocampus (Penfield \& Milner, 1958). The operated controls showed no evidence of structural damage.

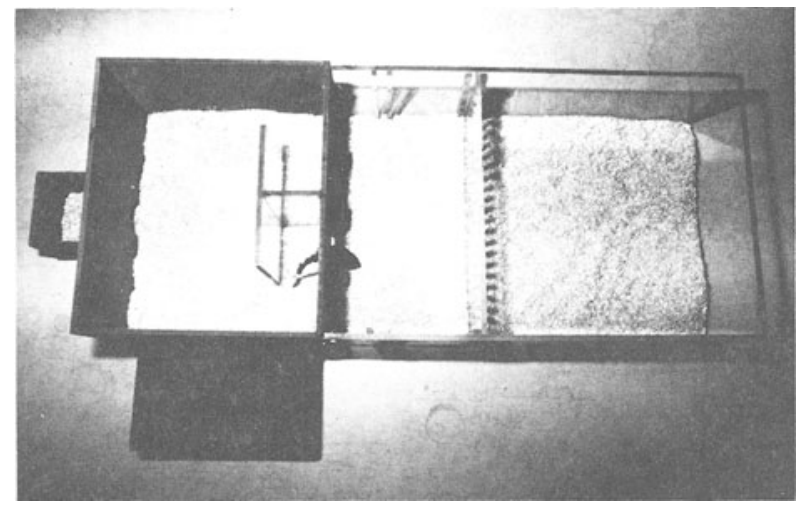

Figure 1. Homing apparatus.

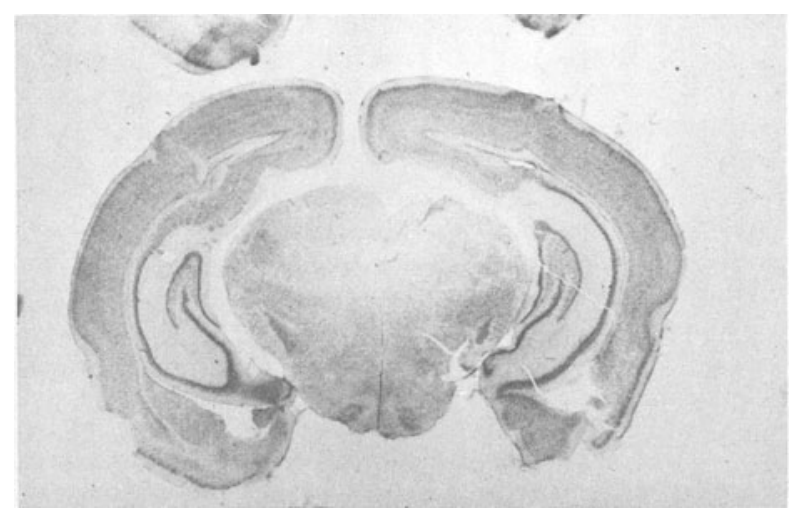

Figure 2. Radio frequency lesions of the entorhinal area in a 1-day-old rat.

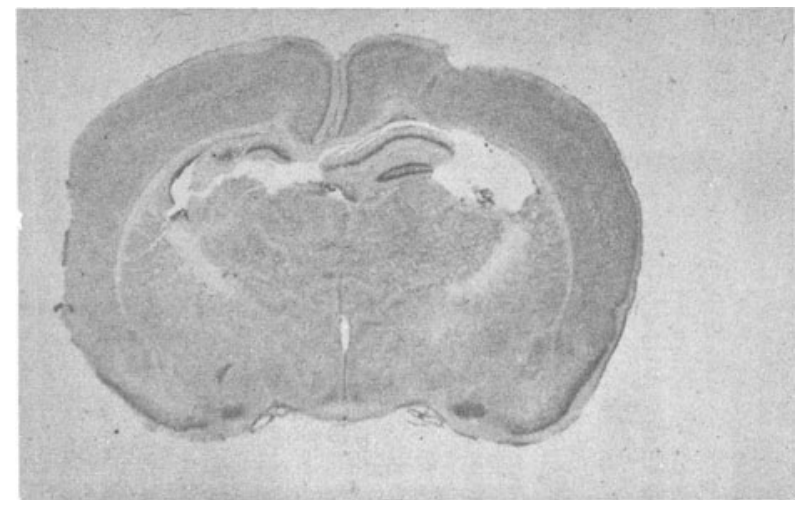

Figure 3. Radio frequency lesions of the hippocampus in a 1-day-old rat.

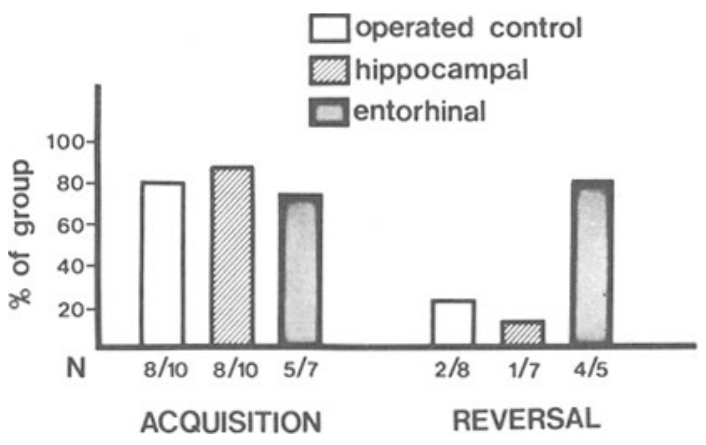

Figure 4. Proportion of pups reaching criterion.

\section{RESULTS}

A Fisher exact probability test did not show statistically significant differences between the number of control and experimental animals that reached criterion on the acquisition task. When tested on the reversal, a significantly larger proportion of animals in the entorhinal group reached criterion than did those in the hippocampal and operated control groups combined (Figure 4). This difference was significant at the .03 level of confidence (two-tailed). There were no statistically significant differences, however, between the entorhinal and control groups or between the hippocampal and control groups.

\section{DISCUSSION}

Of striking interest is the fact that a statistically significantly larger number of pups with entorhinal ablations than pups with hippocampal ablation reversed in the homing left-right spatial reversal tasks. This may be due in part to the finding reported on numerous occasions (Loesche \& Steward, 1977; Lynch, Gall, Rose, \& Cotman, 1976; Zimmer, 1974) that lesions caused a proliferation of fibers from the contralateral entorhinal cortex. In the adult, stimulation of the remaining intact entorhinal area results in a short latency activation and discharge in the granule cell layer of the contralateral dentate gyrus. This suggests that the reinnervated dentate gyrus of the hippocampus is electrophysiologically functional. As a result of possible regrowth from the contralateral undamaged entorhinal area, the infant rats in the present study performed better as a whole than the hippocampal and operated controls combined. Surgery took place prior to testing with enough time for the reinnervation to occur. What is more interesting is that pups with bilateral lesions of the entorhinal area were facilitated on the reversal task. This finding supports earlier studies on monkeys (Zola \& Mahut, 1973). It is possible that partial deefferentation of the hippocampus may result in paradoxically facilitated performance on selected tasks in both rats and monkeys, provided that lesions are made prior to hippocampal development and that animals are not handicapped during initial discrimination training (Gazzara \& Altman, 1981).

In summary, radio frequency lesions in the hippocampal formation of 1-day-old rats produced facilitation of reversal performance on a left-right spatial discrimination task. The present study also provides support for the earlier finding with monkeys that lesions of the hippocampus can produce facilitatory rather than deleterious effects. Behavioral deficits may occur only if lesions are made after the hippocampus has completed a certain amount of development. 


\section{REFERENCES}

BAYER, S. A. Development of the hippocampal region in the rat. I: Neurogenesis examined with $3 \mathrm{H}$-thymidine autoradiography. Journal of Comparative Neurology, 1980, 190, 87-114.

Bulut, F. G., \& Altman, J. Spatial and tactile discrimination learning in infant rats motivated by homing. Developmental Psychobiology, 1974, 7, 465-473.

Gazzara, R. A., \& Altman, J. Early postnatal X-irradiation of the hippocampus and discrimination learning in adult rats. Journal of Comparative and Physiological Psychology, 1981, 95, 484-495.

Hebs, D. O., \& Penfield, W. Human behavior after extensive bilateral removal from the frontal lobes. Archives of Neurology and Psychiatry, 1940, 44, 421-438.

Loesche, J., \& STEWARD, O. Behavioral correlates of denervation and reinnervation of the hippocampal formation of the rat: Recovery of alternation performance following unilateral entorhinal cortex lesions. Brain Research Bulletin, 1977, 2, 31-39.

Lynch, G., Gall, R., Rose, G., \& Cotman, C. Changes in the distribution of the dentate gyrus associational system following unilateral and bilateral entorhinal lesions in the adult rat. Brain Research, 1976, 110, 57-71.

Milner, B. Hemispheric specialization: Scope and limits in F. O. Schmitt \& F. G. Worden (Eds.), The neuroscience third study program. Cambridge, Mass: M.I.T. Press, 1974.

Penfield, W., \& Milner, B. Memory deficit produced by bilateral lesions in the hippocampal zone. Archives of Neurology and Psychiatry, 1958, 79, 475-497.

Schwartz, S. Effects of neonatal cortical lesions and early environmental factors on adult rat behavior. Journal of Comparative and Physiological Psychology, 1963, 57, 72-77.

Teuber, H. L. Mental retardation after early trauma to the brain: Some issues in search of facts. In C. R. Angle \& E. A. Bering, Jr. (Eds.), Physical trauma as an etiological agent in mental retardation. Bethesda, Md: National Institutes of Health, 1971.

Zimmer, J. Proximity as a factor in the regulation of aberrant growth in postnatally de-afferentated fascia dentata. Brain Research, 1974, 72, 137-142.

ZolA, S., \& MAHUT, H. Paradoxical facilitation of object reversal learning after transaction of the fornix in monkeys. Neuropsychologia, 1973, 11, 271-284. 